\title{
Infinite soft theorems from gauge symmetry
}

\author{
Zhi-Zhong Li, ${ }^{*}$ Hung-Hwa Lin, ${ }^{\dagger}$ and Shun-Qing Zhang \\ Department of Physics and Center for Theoretical Sciences, \\ National Taiwan University, Taipei 10617, Taiwan
}

(Received 7 March 2018; published 6 August 2018)

\begin{abstract}
In this paper, we show that the soft behavior of photons and graviton amplitudes, after projection, can be determined to infinite order in soft expansion via ordinary on-shell gauge invariance. In particular, as one of the particle's momenta becomes soft, gauge invariance relates the nonsingular diagrams of an $n$-point amplitude to that of the singular ones up to possible homogeneous terms. We demonstrate that with a particular projection of the soft limit the homogeneous terms do not contribute, and one arrives at an infinite soft theorem. This reproduces the result recently derived from the Ward identity of large gauge transformations. We also discuss the modification of these soft theorems due to the presence of higher-dimensional operators.
\end{abstract}

DOI: 10.1103/PhysRevD.98.045004

\section{INTRODUCTION}

It has long been known that on-shell gauge invariance can be utilized to obtain universal soft behaviors of scattering amplitudes for photons and gravitons. Gauge invariance dictates that the amplitude must vanish when one of its polarization vectors/tensors is replaced by the momenta. Taking one of the momenta of an $n$-point amplitude $\left(M_{n}\right)$ to be soft, the gauge invariance of the soft leg then relates the finite part of the amplitude to the singular diagrams, which is given by the product of a threepoint vertex and the $n-1$-point amplitude. The latter is then amenable to the form of a "soft operator" acting upon the $n-1$-point amplitude. Thus, one schematically has

$$
\left.M_{n}\right|_{q \rightarrow 0}=\sum_{i=-1}^{a}\left(\mathcal{S}_{i}\right) M_{n-1}+\mathcal{O}\left(q^{a+1}\right),
$$

where $q$ is the soft momenta and $\mathcal{S}_{i}$ are the soft operators with the subscript $i$ indicating to which degree in the $q$ expansion is it defined. For photons, $a=0$, while for gravitons, $a=1$ [1-4]. The reason why the soft theorem always terminates at a finite order is because when using gauge invariance one can only determine the finite part of the amplitude up to a homogeneous solution, denoted as

\footnotetext{
*b02202003@ntu.edu.tw

†hunghwalin@gmail.com

*24019025@gmail.com
}

Published by the American Physical Society under the terms of the Creative Commons Attribution 4.0 International license. Further distribution of this work must maintain attribution to the author(s) and the published article's title, journal citation, and DOI. Funded by SCOAP ${ }^{3}$.
$R^{\mu}$, satisfying $q \cdot R=0$ for which one has no control. From the general principle of locality and Lorentz symmetry, one can only determine this term up to a certain order, which sets $a$.

A few years ago, Strominger and Cachazo $[5,6]$ demonstrated that the soft theorems for gravitons can alternatively be interpreted as a consequence of extended Bondi, van der Burg, Metzner, and Sachs symmetry [7,8]. This generated new interest in soft theorems of amplitudes and their relationship with underlying symmetries. As the new interpretation only relies on the structure of space-time at asymptotic infinity, it can be viewed as a direct constraint on any theory of quantum gravity that admits asymptotic flat solutions. However, given that the resulting soft theorems can be derived via ordinary gauge symmetry, it is natural to ask, in the context of amplitudes, what precisely the new interpretation buys us. This is especially intriguing given that the soft theorems are modified at loop level $[9,10]$ as well as by higher-dimensional operators [11,12], the latter of which are tied to the details of the interaction.

Recently, an interesting opportunity presented itself in the form of an infinite-order soft theorem derived from the Ward identity of large gauge transformations ${ }^{1}$ by Hamada and Shiu [14]. An interesting feature of the newly derived soft theorem is that it only gives the soft limit of the projected piece of the amplitude. For example, for photons, one has

$$
\left.\Omega_{\mu \alpha_{1} \cdots \alpha_{l}} \partial_{q^{\alpha_{1}}} \cdots \partial_{q^{\alpha_{l}}} M_{n}^{\mu}\right|_{q \rightarrow 0}=\sum_{i=-1}^{\infty}\left(\mathcal{S}_{i, \nu}^{\mu}\right) M_{n-1}^{\nu}
$$

\footnotetext{
${ }^{1}$ See also Ref. [13] for the derivation of a Ward identity for residual gauge symmetry.
} 
where $M_{n}^{\mu}$ is the amplitude with one of the polarization vectors $\epsilon^{\mu}$ stripped off and $\Omega_{\mu \alpha_{1} \cdots \alpha_{l}}$ is a symmetric tensor. ${ }^{2}$

In this paper, we will show that the above can again be derived by ordinary on-shell gauge invariance. Recall that the derivation based on gauge symmetry yields soft theorems at finite order due to the potential ambiguities, i.e., the aforementioned $R^{\mu}$. We will demonstrate that such terms vanish upon the projection. In other words, the infinite-order soft theorem derived in Ref. [14] is precisely the part of the amplitude that is completely determined by ordinary gauge symmetry. We will demonstrate this for photons and gravitons. Furthermore, we will use explicit examples to demonstrate that, while $R^{\mu}$ can be projected out, it is nonetheless not zero. Finally, for completeness, we will discuss the modification of this infinite soft theorem by the presence of higher-dimensional operators.

\section{SOFT THEOREM FROM WARD IDENTITY}

We follow Refs. $[15,16]$ to investigate infinite-order soft limits of photon and graviton amplitude using ordinary onshell gauge invariance. Beyond the usual (sub)subleading soft theorems, they could only be fixed up to a homogeneous term. However, if restrict our attention to certain projected pieces of the amplitude, such a term does not contribute, and soft theorems can be obtained up to infinite order. For photons, we reproduce the result from large gauge transformations [14]. For gravitons, our result is more general, in that it gives the soft limit of a broader piece of the amplitude. That is, the soft theorems here left fewer undetermined pieces than the result in Ref. [14].

\section{A. Photon soft theorem}

Consider a scattering amplitude,

$$
M_{n+m+1}\left(q ; p_{1}, \ldots, p_{m}, k_{1}, \cdots k_{n}\right),
$$

involving one soft photon, $n$ hard photons, and $m$ matter scalars, with momenta $q, k_{1}, \cdots k_{n}$, and $p_{1}, \ldots, p_{m}$, respectively. Since the amplitude is a linear function in polarization vectors, it can be expressed as

$$
M_{n+m+1}=\epsilon_{q, \mu} M_{n+m+1}^{\mu},
$$

where $\epsilon_{q}$ is the polarization vector for the soft photon. In the following, we discuss the partial amplitude $M_{n+m+1}^{\mu}$ without the polarization vector.

The scattering amplitude contains a contribution with a pole in the soft momentum $q$ and those with no pole, as in Fig. 1,

\footnotetext{
${ }^{2}$ In Ref. [14], the tensor is symmetric traceless. However, the trace piece automatically vanishes upon contracting with the polarization vectors, as we will discuss shortly, and thus does not make a difference.
}

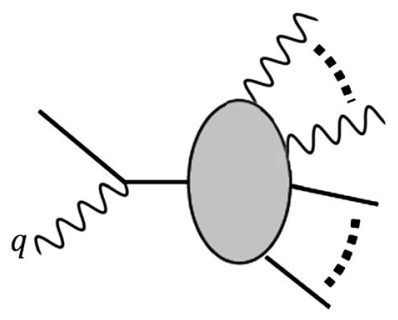

(a)

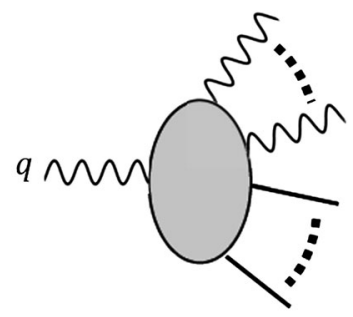

(b)
FIG. 1. Contributions (a) with pole and (b) without pole.

$$
\begin{aligned}
& M_{n+m+1}^{\mu}\left(q ; p_{1}, \ldots, p_{m}, k_{1}, \cdots k_{n}\right) \\
& \quad=\sum_{i=1}^{m} e_{i} \frac{p_{i}^{\mu}}{p_{i} \cdot q} M_{n+m}\left(p_{1}, \ldots, p_{i}+q, \ldots, p_{m}, k_{1}, \cdots k_{n}\right) \\
& \quad+N^{\mu}\left(q ; p_{1}, \ldots, p_{m}, k_{1}, \cdots k_{n}\right),
\end{aligned}
$$

where $e_{i}$ are the charges of scalars, $N^{\mu}$ denotes the terms without a pole, and $M_{n+m}$ denotes the lower-point amplitude without the soft photon. The pole terms can only arise from the three-point vertex involving the soft photon and an external scalar, since there are no self-interactions for photons. At leading order, there is no contribution from $N^{\mu}$, giving the leading soft theorem

$$
\left.M_{n+m+1}^{\mu}\right|_{q \rightarrow 0}=\sum_{i=1}^{m} e_{i} \frac{p_{i}^{\mu}}{p_{i} \cdot q} M_{n+m}+\mathcal{O}\left(q^{0}\right) .
$$

Beyond this order, $N^{\mu}$ must be considered.

On-shell gauge invariance relates $N^{\mu}$ to the lower-point amplitude $M_{n+m}$ by dictating

$$
0=q_{\mu} M_{n+m+1}^{\mu}=\sum_{i=1}^{m} e_{i} M_{n+m}+q_{\mu} N^{\mu} .
$$

At zeroth order, the constraint gives charge conservation,

$$
\sum_{i=1}^{m} e_{i}=0
$$

Beyond zeroth order, we may expand $N^{\mu}$ as

$$
N^{\mu}=\sum_{l} q_{\alpha_{1}} \cdots q_{\alpha_{l}} N_{l}^{\mu, \alpha_{1} \cdots \alpha_{l}}
$$

since it is polynomial in $q$ at tree level. Then, order by order, we have

$$
\begin{aligned}
& q_{\mu} q_{\alpha_{1}} \cdots q_{\alpha_{l}} \\
& \quad \times\left(\sum_{i=1}^{m} \frac{e_{i}}{(l+1) !} \partial_{i}^{\mu} \partial_{i}^{\alpha_{1}} \cdots \partial_{i}^{\alpha_{l}} M_{n}+N_{l}^{\mu, \alpha_{1} \cdots \alpha_{l}}\right)=0
\end{aligned}
$$

so that $N_{l}^{\mu}$ can be expressed in terms of $M_{n+m}$ up to a homogeneous term $R_{l}$, 


$$
N_{l}^{\mu, \alpha_{1} \cdots \alpha_{l}}=-\sum_{i=1}^{m} \frac{e_{i}}{(l+1) !} \partial_{i}^{\mu} \partial_{i}^{\alpha_{1}} \cdots \partial_{i}^{\alpha_{l}} M_{n+m}+R_{l}^{\mu \alpha_{1} \cdots \alpha_{l}}
$$

where $R_{l}$ satisfies the Ward identity by itself,

$$
q_{\mu} q_{\alpha_{1}} \cdots q_{\alpha_{l}} R_{l}^{\mu \alpha_{1} \cdots \alpha_{l}}=0
$$

posing as an ambiguous term. Generally, $R_{l}$ can be separated into three pieces,

$$
R^{\mu \alpha_{1} \cdots \alpha_{l}}=T_{l}^{\mu \alpha_{1} \cdots \alpha_{l}}+O_{l}^{\mu \alpha_{1} \cdots \alpha_{l}}+A_{l}^{\mu \alpha_{1} \cdots \alpha_{l}}
$$

where $T$ is the trace part,

$$
T_{l}^{\mu \alpha_{1} \cdots \alpha_{l}}=\eta^{\left(\mu \alpha_{1}\right.} B_{l}^{\left.\alpha_{2} \cdots \alpha_{l}\right)}
$$

$O$ is the symmetric traceless part satisfying

$$
\eta_{\mu \alpha_{i}} O_{l}^{\mu \alpha_{1} \cdots \alpha_{l}}=\eta_{\alpha_{i} \alpha_{j}} O_{l}^{\mu \alpha_{1} \cdots \alpha_{l}}=0, \quad \text { for any } i, j
$$

and $A$ contains the remaining terms, which are antisymmetric in any two indices among $\mu$ and $\alpha$ 's. Since any arbitrary $A$ or $T$ automatically satisfies Eq. (12), the symmetric traceless part $O$ must satisfy Eq. (12) by itself. It is then straightforward to show that $O$ must vanish. ${ }^{3}$ The trace part $T$ can also be discarded, since the contribution of $R_{l}$ to $N^{\mu}$ is in the form of

$$
q_{\alpha_{1}} \cdots q_{\alpha_{l}} R_{l}^{\mu \alpha_{1} \cdots \alpha_{l}}
$$

so that $T$ produces either terms with $q^{2}=0$ for massless $q$ or $q^{\mu}$, which vanishes after putting back the polarization vector of the soft photon, as in Eq. (4). Therefore, only the antisymmetric part needs to be considered, giving us

$$
N_{l}^{\mu, \alpha_{1} \cdots \alpha_{l}}=-\sum_{i=1}^{m} \frac{e_{i}}{(l+1) !} \partial_{i}^{\mu} \partial_{i}^{\alpha_{1}} \cdots \partial_{i}^{\alpha_{l}} M_{n+m}+A_{l}^{\mu \alpha_{1} \cdots \alpha_{l}}
$$

Plugging this into the expression for full amplitude Eq. (5), we get an incomplete soft theorem for all orders up to the antisymmetric homogeneous term $A$,

$$
\begin{aligned}
M_{n+m+1,(l)}^{\mu}= & \sum_{i=1}^{m} \frac{1}{(l+1) !} \frac{e_{i}}{p_{i} \cdot q} q_{\nu} J_{i}^{\mu \nu}\left(q \cdot \partial_{i}\right)^{l} M_{n+m} \\
& +q_{\alpha_{1}} \cdots q_{\alpha_{l}} A_{l}^{\mu \alpha_{1} \cdots \alpha_{l}}
\end{aligned}
$$

where

\footnotetext{
${ }^{3}$ For arbitrary $p$, we have following separations: $p_{\mu}=$ $\sum_{i=1}^{3} c_{i} q_{i \mu}$ and $p_{\mu} p_{\nu}=c_{0} \eta_{\mu \nu}+\sum_{i=1}^{3} c_{i} q_{i \mu} q_{i \nu}$, where $q_{i}^{2}=0$.
}

$$
J_{i}^{\mu \nu}=p_{i}^{\mu} \frac{\partial}{\partial p_{i \nu}}-p_{i}^{\nu} \frac{\partial}{\partial p_{i \mu}}
$$

The case $l=0$ contains no homogeneous term, giving us the well-known subleading soft theorem. At higher order, $A$ can be nonzero, but we may single out the piece totally symmetric in $\alpha_{i}$ and $\mu$ by contracting with a totally symmetric tensor $\Omega_{\mu \alpha_{1} \cdots \alpha_{l}}$. A is then removed, giving a partial soft term up to all orders in $q$,

$$
\begin{aligned}
& \left.\Omega_{\mu \alpha_{1} \cdots \alpha_{l}} \partial^{\alpha_{1}} \cdots \partial^{\alpha_{l}} M_{n+m+1}^{\mu}\right|_{q \rightarrow 0} \\
& =\Omega_{\mu \alpha_{1} \cdots \alpha_{l}} \partial^{\alpha_{1}} \cdots \partial^{\alpha_{l}} \\
& \quad \times\left.\left[\sum_{i=1}^{m} \frac{1}{(l+1) !} \frac{e_{i}}{p_{i} \cdot q} q_{\nu} J_{i}^{\mu \nu}\left(q \cdot \partial_{i}\right)^{l} M_{n+m}\right]\right|_{q \rightarrow 0},
\end{aligned}
$$

where we adopt short-hand notation $\partial^{\alpha_{j}}=\partial / \partial q^{\alpha_{j}}$ and $q \cdot \partial_{i}=q \cdot \partial / \partial p_{i}$. These are exactly the infinite-order soft theorems in Ref. [14].

\section{B. Graviton}

The derivation for soft theorems of gravitons is similar, except that Ward identity can be applied twice, pushing the usual soft theorem to subsubleading order and placing more stringent constraints on the homogeneous terms at higher order.

In principle, we should consider a general amplitude involving one soft graviton, $n$ hard gravitons, and $m$ matter scalars,

$$
M_{n+m+1}\left(q, p_{1}, \ldots, p_{m}, k_{1}, \ldots, k_{n}\right)
$$

with momenta $q, k_{1}, \cdots k_{n}$, and $p_{1}, \ldots, p_{m}$, respectively. The pole contribution could then come from both the scalar-graviton vertex and the three-point self-interaction of gravitons. Though the derivation procedure is unchanged, this complicates the calculation of soft factors. For clarity, we separately consider two cases: one involving only a single graviton and one involving multiple gravitons without scalars. The most general soft theorem can be obtained simply by combining the results of the two.

We first discuss the amplitude involving a single soft graviton and $m$ scalars, with momenta $q$ and $p_{1}, \ldots, p_{m}$, respectively,

$$
M_{m+1}\left(q, p_{1}, \ldots, p_{m}\right)
$$

The scattering amplitude again contains contributions with and without a pole in the soft momentum $q$,

$$
M_{m+1}^{\mu \nu}=\sum_{i=1}^{m} \frac{p_{i}^{\mu} p_{i}^{\nu}}{p_{i} \cdot q} M_{m}+N^{\mu \nu}
$$


with $N^{\mu \nu}$ denoting the terms without a pole and $M_{n}$ denoting the lower-point amplitude without the soft graviton. Expanding in the power of soft momentum $q$, only the pole diagrams contribute to the leading piece

$$
M_{(-1)}^{\mu \nu}=\sum_{i=1}^{m} \frac{p_{i}^{\mu} p_{i}^{\nu}}{p_{i} \cdot q}
$$

However, the higher-order pieces contain both pole and gut diagrams, and by Ward identity, parts of gut diagrams relate to the pole ones.

$$
q_{\mu}\left(\sum_{i=1}^{m} \frac{p_{i}^{\mu} p_{i}^{\nu}}{p_{i} \cdot q} M_{m}\left(p_{i}+q\right)+N^{\mu \nu}\right)=0
$$

Expanding $N^{\mu \nu}$ around $q \rightarrow 0$,

$$
N^{\mu \nu}=\sum_{l} q^{\alpha_{1}} \cdots q^{\alpha_{l}} N_{l}^{\mu \nu, \alpha_{1} \cdots \alpha_{l}},
$$

we similarly obtain $N_{l}$ up to a homogeneous term $R_{l}$,

$N_{l}^{\mu \nu, \alpha_{1} \cdots \alpha_{l}}=-\sum_{i=1}^{m} \frac{p_{i}^{\nu}}{(l+1) !} \partial_{i}^{\mu} \partial_{i}^{\alpha_{1}} \cdots \partial_{i}^{\alpha_{l}} M_{m}+R_{l}^{\mu \nu \alpha_{1} \cdots \alpha_{l}}$,

where

$$
q_{\mu} q_{\alpha_{1}} \cdots q_{\alpha_{l}} R_{l}^{\mu \nu \alpha_{1} \cdots \alpha_{l}}=0
$$

Again, $R_{l}$ can be separated into three pieces,

$$
R^{\mu \nu \alpha_{1} \cdots \alpha_{l}}=T_{l}^{\mu \nu \frac{\alpha_{1} \cdots \alpha_{l}}{\omega}}+O_{l}^{\mu \nu \frac{\alpha_{1} \cdots \alpha_{l}}{\omega}}+A_{l}^{\mu \nu \frac{\alpha_{1} \cdots \alpha_{l}}{l}}
$$

where $T$ is the trace part,

$$
T_{l}^{\mu \nu \alpha_{1} \cdots \alpha_{l}}=\eta^{\left(\mu \alpha_{1}\right.} B_{l}^{\left.\alpha_{2} \cdots \alpha_{l}\right) \nu}
$$

$O$ is the symmetric traceless part satisfying

$$
\eta_{\mu \alpha_{i}} O_{l}^{\mu \nu \alpha_{1} \cdots \alpha_{l}}=\eta_{\alpha_{i} \alpha_{j}} O_{l}^{\mu \nu \alpha_{1} \cdots \alpha_{l}}=0, \quad \text { for any } i, j
$$

and $A$ is all terms that are antisymmetric in any two indices among $\mu$ and $\alpha$ 's. For reasons identical to the case for photons, only $A$ survives. Thus, we can rewrite our amplitude in the $l$ th order as ${ }^{4}$

\footnotetext{
${ }^{4}$ Here, we have used $q^{2}=0$ and drop terms proportional to $q^{\mu}$, which would not contribute to the gauge-invariance amplitude.
}

$$
\begin{aligned}
M_{m+1,(l)}^{\mu \nu}= & \sum_{i=1}^{m} \frac{1}{(l+1) !} \frac{p_{i}^{\nu}}{p_{i} \cdot q} \\
& \times\left[p_{i}^{\mu}\left(q \cdot \partial_{i}\right)-\left(p_{i} \cdot q\right) \partial_{i}^{\mu}\right]\left(q \cdot \partial_{i}\right)^{l} M_{m} \\
& +q_{\alpha_{1}} \cdots q_{\alpha_{l}} A \frac{\mu \nu \alpha_{l} \cdots \alpha_{l}}{l} .
\end{aligned}
$$

For $l=0$, we get

$$
M_{n+1,(0)}^{\mu \nu}=\sum_{i=1}^{m} \frac{p_{i}^{\nu}}{k \cdot q} q_{\alpha} J_{i}^{\mu \alpha} M_{m}
$$

but for $l>0$, we need to impose the gauge-invariance condition again,

$$
\begin{aligned}
& q_{\nu}\left(\sum_{i=1}^{m} \frac{1}{(l+1) !} \frac{p_{i}^{\nu}}{p_{i} \cdot q}\left[p_{i}^{\mu}\left(q \cdot \partial_{i}\right)-\left(p_{i} \cdot q\right) \partial_{i}^{\mu}\right]\left(q \cdot \partial_{i}\right)^{l} M_{m}\right. \\
& \left.+q_{\alpha_{1}} \cdots q_{\alpha_{l}} A_{l}^{\mu \nu \alpha \alpha_{1} \cdots \alpha_{l}}\right)=0 .
\end{aligned}
$$

Thus, we get

$$
\begin{aligned}
A_{l}^{\mu \nu} \frac{\alpha_{1} \cdots \alpha_{l}}{}= & -\sum_{i} \frac{1}{(l+1) !}\left(p_{i}^{\mu} \partial_{i}^{\alpha_{1}}-p_{i}^{\alpha_{1}} \partial_{i}^{\mu}\right) \partial_{i}^{\alpha_{2}} \cdots \partial_{i}^{\alpha_{l}} \partial_{i}^{\nu} M_{m} \\
& +C_{l}^{\mu \nu \alpha_{1} \cdots \alpha_{l}} .
\end{aligned}
$$

For similar reasons, $C$ also contains only a trace and antisymmetric part in any two indices among $\nu$ and $\alpha$ 's. Defining $L^{\mu \nu}$ as an antisymmetric tensor in $\mu$ and $\nu$, we can write

$$
\begin{aligned}
C_{l}^{\mu \nu \alpha_{1} \cdots \alpha_{l}}= & \sum_{i, j} L^{\mu \alpha_{i}}\left(L^{\nu \alpha_{j}}+\eta^{\nu \alpha_{j}}\right) D_{l}^{\alpha_{1} \cdots \tilde{\alpha}_{i} \cdots \tilde{\alpha}_{j} \cdots \alpha_{l}} \\
& +L^{\alpha_{i} \alpha_{j}} E_{l}^{\mu \nu \alpha_{1} \cdots \tilde{\alpha}_{i} \cdots \tilde{\alpha}_{j} \cdots \alpha_{l}}
\end{aligned}
$$

so the amplitude becomes ${ }^{5,6}$

$$
\begin{aligned}
M_{m+1,(l)}^{\mu \nu}= & \sum_{i=1}^{m} \frac{1}{(l+1) !} \frac{q_{\alpha} q_{\beta}}{p_{i} \cdot q} J_{i}^{\mu \alpha} J_{i}^{\nu \beta}\left(q \cdot \partial_{i}\right)^{l-1} M_{m} \\
& +q_{\alpha_{1}} \cdots q_{\alpha_{l}} \sum_{i, j} L^{\mu \alpha_{i}} L^{\nu \alpha_{j}} D_{l}^{\alpha_{1} \cdots \tilde{\alpha}_{i} \cdots \tilde{\alpha}_{j} \cdots \alpha_{l}},
\end{aligned}
$$

For $l=1$, we do not have the $D$ terms, so we now get the subsubleading graviton soft theorem

$$
M_{(1)}^{\mu \nu}=\sum_{i=1}^{m} \frac{q_{\alpha} q_{\beta}}{2 k \cdot q} J_{i}^{\mu \alpha} J_{i}^{\nu \beta} .
$$

\footnotetext{
${ }^{5}$ The terms proportional to $q^{\nu}$ or $\eta^{\mu \nu}$ are dropped in the gaugeinvariance amplitude.

${ }^{6} \tilde{\alpha}_{i}$ means the entry $\alpha_{i}$ is removed.
} 
However, for $l>1$, there are $D$ terms not given by the Ward identity:

$$
\begin{aligned}
\partial^{\alpha_{1}} \cdots & \partial^{\alpha_{l}} M_{n+1,(l)}^{\mu \nu} \\
= & \partial^{\alpha_{1}} \cdots \partial^{\alpha_{l}} \\
& \times\left[\sum_{i=1}^{m} \frac{1}{(l+1) !} \frac{q_{\alpha} q_{\beta}}{p_{i} \cdot q} J_{i}^{\mu \alpha} J_{i}^{\nu \beta}\left(q \cdot \partial_{i}\right)^{l-1}\right] M_{n} \\
& +L^{\mu \alpha_{i}} L^{\nu \alpha_{j}} D_{l}^{\alpha_{1} \cdots \tilde{\alpha}_{i} \cdots \tilde{\alpha}_{j} \cdots \alpha_{l}} .
\end{aligned}
$$

Therefore, we can obtain, for example, either pieces symmetric in $\mu$ and $\alpha_{i}$ or those symmetric in $\nu$ and $\alpha_{i}$,

$$
\begin{aligned}
& \left.\Omega_{\mu \alpha_{1} \cdots \alpha_{l}} \partial^{\alpha_{1}} \cdots \partial^{\alpha_{l}} M_{m+1,(l)}^{\mu \nu}\right|_{q \rightarrow 0} \\
& =\Omega_{\mu \alpha_{1} \cdots \alpha_{l}} \partial^{\alpha_{1}} \cdots \partial^{\alpha_{l}} \\
& \quad \times\left.\left[\sum_{i=1}^{m} \frac{1}{(l+1) !} \frac{q_{\alpha} q_{\beta}}{p_{i} \cdot q} J_{i}^{\mu \alpha} J_{i}^{\nu \beta}\left(q \cdot \partial_{i}\right)^{l-1}\right] M_{m}\right|_{q \rightarrow 0} \\
& \left.\Omega_{\nu \alpha_{1} \cdots \alpha_{l}} \partial^{\alpha_{1}} \cdots \partial^{\alpha_{l}} M_{m+1,(l)}^{\mu \nu}\right|_{q \rightarrow 0} \\
& =\Omega_{\nu \alpha_{1} \cdots \alpha_{l}} \partial^{\alpha_{1}} \cdots \partial^{\alpha_{l}} \\
& \quad \times\left.\left[\sum_{i=1}^{m} \frac{1}{(l+1) !} \frac{q_{\alpha} q_{\beta}}{p_{i} \cdot q} J_{i}^{\mu \alpha} J_{i}^{\nu \beta}\left(q \cdot \partial_{i}\right)^{l-1}\right] M_{m}\right|_{q \rightarrow 0},
\end{aligned}
$$

where $\Omega^{\rho \alpha_{1} \cdots \alpha_{l}}$ is a totally symmetric tensor. The soft theorems from large gauge transformations [14], however, only consider a more restrictive piece,

$$
\begin{aligned}
& {\left.\left[\Omega_{\mu\left(\nu \alpha_{1} \cdots \alpha_{l}\right)}+\Omega_{\nu\left(\mu \alpha_{1} \cdots \alpha_{l}\right)}\right] \partial^{\alpha_{1}} \cdots \partial^{\alpha_{l}} M_{m+1,(l)}^{\mu \nu}\right|_{q \rightarrow 0}} \\
& =\left[\Omega_{\mu\left(\nu \alpha_{1} \cdots \alpha_{l}\right)}+\Omega_{\nu\left(\mu \alpha_{1} \cdots \alpha_{l}\right)}\right] \partial^{\alpha_{1}} \cdots \partial^{\alpha_{l}} \\
& \quad \times\left.\left[\sum_{i=1}^{m} \frac{1}{(l+1) !} \frac{q_{\alpha} q_{\beta}}{p_{i} \cdot q} J_{i}^{\mu \alpha} J_{i}^{\nu \beta}\left(q \cdot \partial_{i}\right)^{l-1}\right] M_{m}\right|_{q \rightarrow 0},
\end{aligned}
$$

where $\Omega_{\mu\left(\nu \alpha_{1} \cdots \alpha_{l}\right)}$ is totally symmetric in $\nu, \alpha_{1}, \ldots, \alpha_{l}$ and traceless in all the indices. ${ }^{7}$ This follows from our result but does not represent the most general derivable soft theorems.

To consider an amplitude involving $n+1$ gravitons,

$$
M_{n+1}\left(q, k_{1}, \ldots, k_{n}\right),
$$

we only have to replace the three-point vertex with the graviton self-interaction. The remaining steps are exactly the same. Taking one graviton soft,

$$
M_{n+1}^{\mu \nu}=\prod_{j=1}^{n} \epsilon_{j, \mu_{j}} \epsilon_{j, \nu_{j}} \sum_{i=1}^{n} \frac{V^{\mu \nu \mu_{i} \nu_{i} \alpha \beta}}{k_{i} \cdot q} M_{n, \alpha \beta}^{\mu_{1} \nu_{1} \cdots \tilde{\mu}_{i} \tilde{\nu}_{i} \cdots \mu_{n} \nu_{n}}+N^{\mu \nu},
$$

\footnotetext{
${ }^{7}$ See footnote 2 in the Introduction.
}

where $V$ is the graviton self-interaction vertex,

$$
\begin{aligned}
V^{\mu \nu \mu_{i} \nu_{i} \alpha \beta} & =\left(k_{i}^{\mu} \eta^{\alpha \mu_{i}}+q_{\rho} \Sigma^{\rho \mu \alpha \mu_{i}}\right)\left(k_{i}^{\nu} \eta^{\beta \nu_{i}}+q_{\tau} \Sigma^{\tau \nu \beta \nu_{i}}\right) \\
\Sigma^{a b c d} & =\eta^{a c} \eta^{b d}-\eta^{a d} \eta^{b c} .
\end{aligned}
$$

and $M_{n}$ is the amplitude involving the remaining $n$ gravitons. Again, expanding $N$ in $q$ and applying the Ward identity, we can obtain, for $q^{0}$ order, ${ }^{8}$ the subleading soft theorem,

$$
M_{n+1,(0)}^{\mu \nu}=\sum_{i=1}^{n} \frac{k_{i}^{\nu}}{k \cdot q} q_{\alpha} J_{i}^{\prime \mu \alpha} M_{n}
$$

where

$$
J_{i}^{\prime \mu \nu}=k_{i}^{\mu} \partial_{i}^{\nu}-k_{i}^{\nu} \partial_{i}^{\mu}+\epsilon_{i}^{\mu} \frac{\partial}{\partial \epsilon_{i, \nu}}-\epsilon_{i}^{\nu} \frac{\partial}{\partial \epsilon_{i, \mu}} .
$$

As for the $l$ th order at which $l \geq 1$, the expansion of Eq. (23) is

$$
\begin{aligned}
M_{n+1,(l)}^{\mu \nu}= & \prod_{j=1}^{n} \epsilon_{j, \mu_{j} \nu_{j}} \sum_{i=1}^{n}\left[\frac{k_{i}^{\mu} \eta^{\alpha \mu_{i}} k_{i}^{\nu} \eta^{\beta \nu_{i}}}{k_{i} \cdot q} \frac{\left(q \cdot \partial_{i}\right)^{l+1}}{(l+1) !}\right. \\
& +\frac{k_{i}^{\mu} \eta^{\alpha \mu_{i}} q_{\tau} \Sigma^{\tau \nu \beta \nu_{i}}+k_{i}^{\nu} \eta^{\beta \nu_{i}} q_{\rho} \Sigma^{\mu \alpha \alpha \mu_{i}}}{k_{i} \cdot q} \frac{\left(q \cdot \partial_{i}\right)^{l}}{l !} \\
& \left.+\frac{q_{\rho} \Sigma^{\rho \mu \alpha \mu_{i}} q_{\tau} \Sigma^{\tau \nu \beta \nu_{i}}}{k_{i} \cdot q} \frac{\left(q \cdot \partial_{i}\right)^{l-1}}{(l-1) !}\right] M_{n, \alpha \beta}^{\mu_{1} \nu_{1} \cdots \tilde{\mu}_{i} \tilde{\nu}_{i} \cdots \mu_{n} \nu_{n}} \\
& +q_{\alpha_{1}} \cdots q_{\alpha_{l}} N_{l}^{\mu \nu \alpha_{1} \cdots \alpha_{l}} .
\end{aligned}
$$

Applying the Ward identity as before, the soft theorem is

$$
\begin{aligned}
M_{n+1,(l)}^{\mu \nu}= & \sum_{i=1}^{n} \frac{q_{\alpha} q_{\beta}}{k_{i} \cdot q}\left[\frac{J_{i}^{\mu \alpha} J_{i}^{\nu \beta}}{(l+1) !}+\frac{1}{2} \frac{J_{i}^{\mu \alpha} U_{i}^{\nu \beta}+U_{i}^{\mu \alpha} J_{i}^{\nu \beta}}{l !}\right. \\
& \left.+\frac{1}{2} \frac{U_{i}^{\mu \alpha} U_{i}^{\nu \beta}}{(l-1) !}\right]\left(q \cdot \partial_{i}\right)^{l-1} M_{n} \\
& +q_{\alpha_{1}} \cdots q_{\alpha_{l}} \sum_{i, j} L^{\mu \alpha_{i}} L^{\nu \alpha_{j}} D_{l}^{\alpha_{1} \cdots \tilde{\alpha}_{i} \cdots \tilde{\alpha}_{j} \cdots \alpha_{l}}
\end{aligned}
$$

where

$$
U_{i}^{\mu \nu}=\epsilon_{i}^{\mu} \frac{\partial}{\partial \epsilon_{i, \nu}}-\epsilon_{i}^{\nu} \frac{\partial}{\partial \epsilon_{i, \mu}}
$$

In particular, the subsubleading piece is

$$
M_{n+1,(1)}^{\mu \nu}=\sum_{i=1}^{n} \frac{q_{\alpha} q_{\beta}}{2 k_{i} \cdot q} J_{i}^{\prime \mu \alpha} J_{i}^{\prime \nu \beta}
$$

without ambiguity. For $l>1$, we again have the partially fixed soft theorem up to infinite order.

\footnotetext{
${ }^{8}$ The terms antisymmetric in $\mu$ and $\nu$ are dropped.
} 


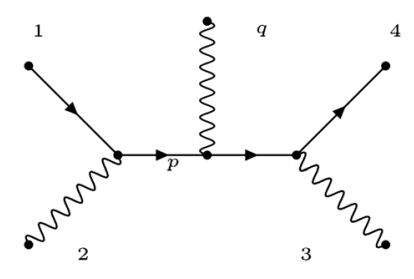

(a)

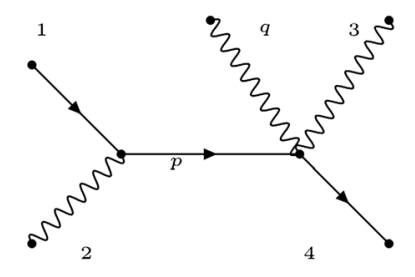

(b)
FIG. 2. No pole diagrams of scalar QED five-point amplitude.

\section{EXAMPLE OF HOMOGENEOUS TERMS}

In the previous section, we showed that one can only have an infinite soft theorem after a projection. Here, we explicitly show the antisymmetric piece of $N_{l}^{\mu, \alpha_{1} \cdots \alpha_{l}}$ that was projected out is indeed nonzero. We use a scalar QED five-point amplitude to demonstrate. The diagrams that contribute to $N$ comes from the soft photon coupled to internal legs as shown in the figure. The contribution from Fig. 2(a) is

$$
\begin{aligned}
N=(i e) & \frac{\left(p_{1}+p\right) \cdot \epsilon_{2}}{\left(p_{1}+p\right)^{2}-m^{2}}\left[(i e)(p+p+q) \cdot \epsilon_{q}\right] \\
& \times(i e) \frac{\left(p+p_{4}+q\right) \cdot \epsilon_{3}}{(p+q)^{2}-m^{2}}+(2 \leftrightarrow 3) \\
= & (i e)^{3} \frac{\left(p_{1}+p\right) \cdot \epsilon_{2}}{\left(p_{1}+p\right)^{2}-m^{2}}\left(2 p \cdot \epsilon_{q}\right) \frac{\left(p+p_{4}+q\right) \cdot \epsilon_{3}}{(p+q)^{2}-m^{2}} \\
& +(2 \leftrightarrow 3)=\epsilon_{q, \mu} N^{\mu},
\end{aligned}
$$

where $p=p_{1}+p_{2}$ and $\mathrm{m}$ is the mass for the scalar. $(2 \leftrightarrow 3)$ means we have to sum over leg- 2 and leg- 3 exchange.

We pull out the polarization vector $\epsilon_{q, \mu}$ and perform a derivative on $N^{\mu}$, then antisymmetrize the $\left(\mu, \alpha_{1}\right)$ index

$$
\begin{aligned}
N^{\mu, \alpha_{1}}= & \frac{\partial}{\partial q_{\alpha_{1}}} N^{\mu} \\
= & (-i e)^{3} \frac{\left(p_{1}+p\right) \cdot \epsilon_{2}}{\left(p_{1}+p\right)^{2}-m^{2}} \frac{\partial}{\partial q^{\alpha_{1}}} \\
& \times\left[(2 p)^{\mu} \frac{\left(p+p_{4}+q\right) \cdot \epsilon_{3}}{(p+q)^{2}-m^{2}}\right] \\
= & (-i e)^{3} \frac{\left(p_{1}+p\right) \cdot \epsilon_{2}}{\left(p_{1}+p\right)^{2}-m^{2}}\left[2 \frac{p^{\mu} \epsilon_{3}^{\alpha_{1}}}{(p+q)^{2}-m^{2}}\right. \\
& \left.+2 \frac{p^{\mu} p^{\alpha_{1}}(p+q) \cdot \epsilon_{3}}{\left[(p+q)^{2}-m^{2}\right]^{2}}\right]=S^{\mu \alpha_{1}}+A^{\mu \alpha_{1}} .
\end{aligned}
$$

The antisymmetric part $A^{\mu \alpha_{1}}$ is not zero.

The contribution from Fig. 2(b) is

$N=(i e) \frac{\left(p_{1}+p\right) \cdot \epsilon_{2}}{\left(p_{1}+p\right)^{2}-m^{2}}\left(-2 i e^{2}\right)\left(\epsilon_{2} \cdot \epsilon_{q}\right)+(2 \leftrightarrow 3)$.
However, this term does not contribute to the $N_{1}^{\mu, \alpha_{1}}$ since it does not involve $q$. After considering Figs. 2(a) and 2(b), we show the antisymmetric part of $N_{1}^{\mu, \alpha_{1}}$ is nonzero, so this term has to be dropped to obtain the infinite soft theorem.

\section{EFFECT OF HIGHER-DIMENSIONAL OPERATORS}

In this section, we consider the infinite soft photon and graviton theorems in the effective field theories. The effective operators start to modify at $q^{0}$ order for the soft photon theorem and $q^{1}$ order for the soft graviton theorem, then continue to affect higher-order ones. We will explicitly show their modifications to the infinite-order soft theorems.

\section{A. Subleading photon soft theorem}

We first look at the modification for the subleading soft photon theorem,

$$
\begin{aligned}
\left.M_{n+m+1}\right|_{q \rightarrow 0}= & \left(q^{-1} \mathcal{S}^{(-1)}+q^{0} \mathcal{S}^{(0)}\right) M_{n+m} \\
& +q^{0} \tilde{\mathcal{S}}^{(0)} \tilde{M}_{n+m}+\mathcal{O}\left(q^{1}\right)
\end{aligned}
$$

where the tilde on the $n$-point amplitudes indicates that the particle type of the $k$ th leg of $\tilde{M}_{n+m}$ may differ from that in $M_{n+m+1}$.

We consider the effect of adding the effective operators $\phi F^{\mu \nu} F_{\mu \nu}$ and $\phi F^{\mu \nu} \tilde{F}_{\mu \nu}$, and then we discuss their corrections to the subleading photon soft theorem.

$$
\text { 1. } \phi \boldsymbol{F}^{\mu \nu} \boldsymbol{F}_{\boldsymbol{\mu \nu}}
$$

When the external leg $q$ is taken soft, the internal $\varphi$ propagator goes on shell, and the amplitude factorizes as shown in Fig. 3.

Its contribution is

$g_{j}\left[\left(k_{j} \cdot q\right)\left(\epsilon_{k_{j}} \cdot \epsilon_{q}\right)-\left(k_{j} \cdot \epsilon_{q}\right)\left(q \cdot \epsilon_{k_{j}}\right)\right] \frac{1}{\left(k_{j} \cdot q\right)} \tilde{M}_{n+m}$

with $g_{j}$ the coupling constant for the three-point vertex.

$$
\text { 2. } \phi \boldsymbol{F}^{\mu \nu} \tilde{\boldsymbol{F}}_{\mu \nu}
$$

We can also consider an effective operator involving the contraction of a dual tensor. Its contribution is

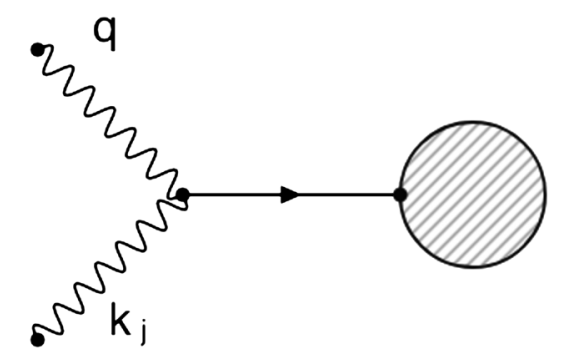

FIG. 3. Pole diagram contribution from $\phi F^{\mu \nu} F_{\mu \nu}$ vertex. 


$$
g_{j}\left[\varepsilon_{\mu \nu \rho \sigma} k_{j}^{\mu} \epsilon_{k_{j}}^{\nu} q^{\rho} \epsilon_{q}^{\sigma}\right] \frac{1}{\left(k_{j} \cdot q\right)} \tilde{M}_{n+m}
$$

where $\varepsilon_{\mu \nu \rho \sigma}$ is the totally antisymmetric rank-4 tensor.

We have shown the effective operator starts making a contribution at subleading $\left(q^{0}\right)$ order, and we now discuss how it affects the infinite soft theorem. Let us consider the $\phi F^{\mu \nu} F_{\mu \nu}$ correction, which has the form of (55). We separate the pole diagrams and the no-pole ones, then see how the Ward identity gives constraints at each $q$ order:

$$
\begin{aligned}
0= & \left.q_{\mu} M_{n+m+1}^{\mu}\right|_{q \rightarrow 0} \\
= & \sum_{i=1}^{m} e_{i} M_{n+m} \\
& +\sum_{j=1}^{n} g_{j}\left[\left(k_{j} \cdot q\right)\left(\epsilon_{k_{j}} \cdot q\right)-\left(k_{j} \cdot q\right)\left(q \cdot \epsilon_{k_{j}}\right)\right] \frac{1}{\left(k_{j} \cdot q\right)} \tilde{M}_{n+m} \\
& +q_{\mu} N^{\mu} \\
= & \sum_{i=1}^{m} e_{i} M_{n+m}+q_{\mu} N^{\mu} .
\end{aligned}
$$

The first term in Eq. (57) is the original pole diagram from photon and matter field coupling, the second one is the pole diagram from the effective operator, and the third one is nopole diagrams. We find that the pole diagram from effective operator does not constrain the no-pole diagram since is gauge invariant itself. Therefore, Eq. (58) is basically the same as Eq. (7). The effective operators do not constrain the form of $N$, but they still modify the infinite soft theorem to be

$$
\begin{aligned}
& \left.\Omega_{\mu \alpha_{1} \cdots \alpha_{l}} \partial^{\alpha_{1}} \cdots \partial^{\alpha_{l}} M_{n+m+1}^{\mu}\right|_{q \rightarrow 0} \\
& =\Omega_{\mu \alpha_{1} \cdots \alpha_{l}}\left\{\sum_{i=1}^{m} \frac{1}{(l+1) !} \frac{e_{i}}{p_{i} \cdot q} q_{\nu} J_{i}^{\mu \nu} \partial_{i}^{\alpha_{1}} \cdots \partial_{i}^{\alpha_{l}} M_{n+m}\right. \\
& \quad+\sum_{j=1}^{n} g\left[\left(k_{j} \cdot q\right) \epsilon_{k_{j}}^{\mu}-\left(q \cdot \epsilon_{k_{j}}\right) k_{j}^{\mu}\right] \\
& \left.\left.\quad \times \frac{1}{\left(k_{j} \cdot q\right)} \partial_{i}^{\alpha_{1}} \cdots \partial_{i}^{\alpha_{l}} \tilde{M}_{n+m}\right]\right\} .
\end{aligned}
$$

The situation is similar for adding the operator $\phi F^{\mu \nu} \tilde{F}_{\mu \nu}$ since this term is also gauge invariant. The infinite soft theorem can be obtained from replacing $\left[\left(k_{j} \cdot q\right) \epsilon_{k_{j}}^{\mu}-\right.$ $\left.\left(q \cdot \epsilon_{k_{j}}\right) k_{j}^{\mu}\right]$ by $\left[\varepsilon_{\mu \nu \rho \sigma} k_{j}^{\mu} \epsilon_{k_{j}}^{\nu} q^{\rho} \epsilon_{q}^{\sigma}\right]$ in the last term of (59).

\section{B. Subsubleading graviton soft theorem}

We can also discuss the subsubleading $\left(q^{1}\right)$ graviton soft theorem correction in the presence of higher-dimensional operators:

$$
\begin{aligned}
\left.M_{n+m+1}\right|_{q \rightarrow 0}= & \left(q^{-1} \mathcal{S}^{(-1)}+q^{0} \mathcal{S}^{(0)}+q^{1} \mathcal{S}^{(1)}\right) M_{n+m} \\
& +q^{1} \tilde{\mathcal{S}}^{(1)} \tilde{M}_{n+m}+\mathcal{O}\left(q^{2}\right) .
\end{aligned}
$$

Let us consider the effect of adding the operator $\phi R_{\sigma \mu \nu}^{\rho} R_{\rho}^{\sigma \mu \nu}$. First, we give the explicit forms of $R_{\sigma \mu \nu}^{\rho}$ and $\Gamma_{\mu \nu}^{\lambda}$,

$$
\begin{aligned}
R_{\sigma \mu \nu}^{\rho} & =\partial_{\mu} \Gamma_{\nu \sigma}^{\rho}-\partial_{\nu} \Gamma_{\mu \sigma}^{\rho}+\Gamma_{\mu \lambda}^{\rho} \Gamma_{\nu \sigma}^{\lambda}-\Gamma_{\nu \lambda}^{\rho} \Gamma_{\mu \sigma}^{\lambda} \\
\Gamma_{\mu \nu}^{\lambda} & =\frac{1}{2} g^{\lambda \sigma}\left(\partial_{\mu} g_{\nu \sigma}+\partial_{\nu} g_{\sigma \mu}-\partial_{\sigma} g_{\mu \nu}\right) .
\end{aligned}
$$

We write $g_{\mu \nu}=\eta_{\mu \nu}+h_{\mu \nu}$, where $h$ is the graviton field. Then, we calculate $\phi R_{\sigma \mu \nu}^{\rho} R_{\rho}^{\sigma \mu \nu}$ for the first order in $h$,

$$
\begin{aligned}
& \left(\frac{1}{2} \eta_{\rho \lambda} \partial^{\mu}\left(\partial^{\nu} h_{q}^{\sigma \lambda}+\partial^{\sigma} h_{q}^{\lambda \nu}-\partial^{\lambda} h_{q}^{\nu \sigma}\right)-(\mu \leftrightarrow \nu)\right) \\
& \quad\left(\frac{1}{2} \eta^{\rho \lambda} \partial_{\mu}\left(\partial_{\nu} h_{k_{i}, \sigma \lambda}+\partial_{\sigma} h_{k_{i}, \lambda \nu}-\partial_{\lambda} h_{k_{i}, \nu \sigma}\right)-(\mu \leftrightarrow \nu)\right) .
\end{aligned}
$$

The subsubleading soft correction due to the above vertex is

$$
q_{\mu} q_{\sigma} E_{q, \lambda \nu}\left(k_{i}^{[\mu} E_{k_{i}}^{\nu][\lambda} k_{i}^{\sigma]}\right) \frac{1}{\left(k_{j} \cdot q\right)} \tilde{M}_{n+m}
$$

where $E_{q, \lambda \nu}$ is the polarization tensor of the soft graviton.

The effective operator we add is by itself gauge invariant, as one can substitute $h_{q, \lambda \nu}$ by $q_{\lambda} \Lambda_{\nu}$ in (63) and find it vanishes. Therefore, it does not constrain the form of the no-pole diagram. The infinite soft graviton soft theorem in the presence of the operator $\phi R_{\sigma \mu \nu}^{\rho} R_{\rho}^{\sigma \mu \nu}$ is

$$
\begin{aligned}
& {\left.\left[\Omega_{\mu\left(\nu \alpha_{1} \cdots \alpha_{l}\right)}+\Omega_{\nu\left(\mu \alpha_{1} \cdots \alpha_{l}\right)}\right] \partial^{\alpha_{1}} \cdots \partial^{\alpha_{l}} M_{m+n+1,(l)}^{\mu \nu}\right|_{q \rightarrow 0}} \\
& =\left[\Omega_{\mu\left(\nu \alpha_{1} \cdots \alpha_{l}\right)}+\Omega_{\nu\left(\mu \alpha_{1} \cdots \alpha_{l}\right)}\right] \partial^{\alpha_{1}} \cdots \partial^{\alpha_{l}} \\
& \quad \times\left\{\left[\sum_{i=1}^{m} \frac{1}{(l+1) !} \frac{q_{\alpha} q_{\beta}}{p_{i} \cdot q} J_{i}^{\mu \alpha} J_{i}^{\nu \beta}\left(q \cdot \partial_{i}\right)^{l-1}\right] M_{m+n}\right. \\
& \left.\quad+\sum_{j=1}^{n} g_{j} \frac{q_{\lambda} q_{\sigma}}{k_{j} \cdot q}\left(k_{i}^{[\lambda} E_{k_{i}}^{\nu][\mu} k_{i}^{\sigma]}\right)\left(q \cdot \partial_{j}\right)^{l-1} \tilde{M}_{n+m}\right\} .
\end{aligned}
$$

One can consider adding the operator $R_{\rho \sigma \mu \nu} F^{\rho \sigma} F^{\mu \nu}$, which is also gauge invariant. The infinite soft theorem for adding this operator has the same expression as (64) except for replacing $k_{i}^{[\lambda} E_{k_{i}}^{\nu][\mu} k_{i}^{\sigma]}$ by $k_{i}^{[\lambda} \epsilon_{k_{i}}^{\nu]} k_{i}^{[\sigma} \epsilon_{k_{i}^{\prime}}^{\mu]}$ in the last term, where $\epsilon_{k_{i}}$ and $\epsilon_{k_{i}^{\prime}}$ are the polarization vectors of the photon.

\section{CONCLUSION AND DISCUSSION}

In this paper, we demonstrate how on-shell gauge invariance can fix the higher-order soft limit of photons and gravitons up to an undetermined homogeneous 
term $R^{\mu}$. This leads to infinite-order soft theorems on certain projected pieces of amplitude, to which the homogeneous term does not contribute. We explicitly worked out the appropriate projection to obtain such pieces and showed that the infinite-order soft theorems derived from large gauge transformations can be completely reproduced here. For the case of gravitons, the theorems derived here are actually more complete, leaving fewer undetermined pieces in the amplitude.

We use explicit examples to demonstrate that the homogeneous term in $R^{\mu}$ can be projected out but can be nonzero, which means we indeed drop some pieces to obtain the infinite-order soft theorem. Finally, we consider the effect of an adding higher-dimensional operator, which starts to modify the photon soft theorem at subleading order and graviton soft theorem at subsubleading order. Moreover, its modification to the infinite-order soft theorem can also be obtained.

The fact that the soft theorems derived from residual gauge symmetries, so far, can all be reproduced by ordinary on-shell gauge symmetry, leaving us asking what the relevance of this new symmetry is on a physical observable like the $\mathrm{S}$ matrix. A pessimist may say that the evidence so far is that there is no relevance beyond that implied by ordinary gauge symmetry, which in a sense is not surprising, given that one projects the correlation function to obtain the S matrix and thus certain information might be projected out. Alternatively, one might say that the symmetry is, in fact, telling us that we are using the wrong asymptotic states for the $\mathrm{S}$ matrix and thus ignorant of its features.
We choose single-particle states for the $\mathrm{S}$ matrix since they are irreducible representations of the Poincare group. This statement makes no distinction between massless and massive kinematics. However, for massless-particle kinematics, it is well known that single particle states are ill defined, since there are no quantum numbers available for us to differentiate colinear multiparticle states. This problem manifests itself in the IR divergence of massless scattering amplitudes. Thus, perhaps the infinite residual gauge symmetry is telling us that the correct asymptotic state for massless kinematics should form a representation of this infinite group. Indeed, recent analysis along this line for QED has demonstrated that this indeed appears to be the case [17], albeit a similar analysis for gravity is still lacking. It will be interesting to understand this in full generality and illustrate how modifications of the three-point interaction via higher-dimension operators changes the conclusion.

Besides single soft theorems discussed here, one may apply the method in Ref. [18] to consider double soft theorems, which involve two, instead of one, soft gauge bosons. It would be interesting to see whether such theorems can be similarly pushed to infinite order by considering a projected piece of the amplitude.

\section{ACKNOWLEDGMENTS}

We thank Yu-tin Huang for suggesting the problem and helping with the draft. Zhi-Zhong Li, Hung-Hwa Lin, and Shun-Qing Zhang are supported by MoST Grant No. 1062628-M-002-012-MY3.
[1] F. E. Low, Phys. Rev. 96, 1428 (1954); M. Gell-Mann and M. L. Goldberger, Phys. Rev. 96, 1433 (1954); S. Saito, Phys. Rev. 184, 1894 (1969).

[2] F. E. Low, Phys. Rev. 110, 974 (1958).

[3] S. Weinberg, Phys. Rev. 135, B1049 (1964); Phys. Rev. 140, B516 (1965).

[4] T. H. Burnett and N. M. Kroll, Phys. Rev. Lett. 20, 86 (1968); J. S. Bell and R. Van Royen, Nuovo Cimento A 60, 62 (1969); V. Del Duca, Nucl. Phys. B345, 369 (1990).

[5] A. Strominger, J. High Energy Phys. 07 (2014) 152; T. He, V. Lysov, P. Mitra, and A. Strominger, J. High Energy Phys. 05 (2015) 151; D. Kapec, V. Lysov, S. Pasterski, and A. Strominger, J. High Energy Phys. 08 (2014) 058.

[6] F. Cachazo and A. Strominger, arXiv:1404.4091.

[7] H. Bondi, M. G. J. van der Burg, and A. W. K. Metzner, Proc. R. Soc. A 269, 21 (1962); R. K. Sachs, Proc. R. Soc. A 270, 103 (1962).

[8] G. Barnich and C. Troessaert, Phys. Rev. Lett. 105, 111103 (2010); J. High Energy Phys. 12 (2011) 105; J. High Energy Phys. 11 (2013) 003.
[9] Z. Bern, S. Davies, and J. Nohle, Phys. Rev. D 90, 085015 (2014).

[10] S. He, Y.t. Huang, and C. Wen, J. High Energy Phys. 12 (2014) 115.

[11] M. Bianchi, S. He, Y.t. Huang, and C. Wen, Phys. Rev. D 92, 065022 (2015).

[12] H. Elvang, C. R. T. Jones, and S. G. Naculich, Phys. Rev. Lett. 118, 231601 (2017).

[13] S. G. Avery and B. U. W. Schwab, J. High Energy Phys. 02 (2016) 031.

[14] Y. Hamada and G. Shiu, Phys. Rev. Lett. 120, 201601 (2018).

[15] Z. Bern, S. Davies, P. Di Vecchia, and J. Nohle, Phys. Rev. D 90, 084035 (2014).

[16] J. Broedel, M. Leeuw, J. Plefka, and M. Rosso, Phys. Rev. D 90, 065024 (2014).

[17] D. Kapec, M. Perry, A. M. Raclariu, and A. Strominger, Phys. Rev. D 96, 085002 (2017).

[18] Z. Z. Li, H. H. Lin, and S. Q. Zhang, J. High Energy Phys. 12 (2017) 32. 